Communications in Physics, Vol. 24, No. 3S2 (2014), pp. 8-12

DOI:10.15625/0868-3166/24/3S2/5014

\title{
STOPPING POWERS OF ALPHA PARTICLES IN SOME GASES AT ENERGIES FROM 1.0 TO 6 MEV
}

\author{
LE HONG KHIEM AND TRAN DINH TRONG \\ Institute of Physics, Vietnam Academy of Science and Technology \\ NGUYEN THE NGHIA \\ Department of Nuclear Physics, Faculty of Physics, Hanoi University of Science, \\ Vietnam National University, Hanoi, Vietnam \\ E-mail:1hkiem@iop.vast.ac.vn
}

Received 20 June 2014

Accepted for publication 20 August 2014

\begin{abstract}
Stopping powers of $\alpha$-particles emitted by ${ }^{148} \mathrm{Gd},{ }^{241} \mathrm{Am}$ and ${ }^{243} \mathrm{Cm}$ isotopes in PR10 and isobutane $\left(\mathrm{C}_{4} \mathrm{H}_{10}+\mathrm{Ar}\right)$ gases have been experimentally measured in the energy region from 1.0 to $5.5 \mathrm{MeV}$. The experimental data were compared with those calculated by SRIM-2013 computer code. It was concluded that the experimental stopping powers in our investigated region are in agreement with the calculated values.
\end{abstract}

Keywords: stopping powers, energy loss, $\alpha$-particle.

\section{INTRODUCTION}

Stopping power of charged particles in different absorbers is an important quantity in many fields of nuclear physics and applications, health physics, radiobiology and other related sciences. The Bethe theory for stopping power has been used for calculating the stopping powers. Owing to its importance, the experimental values of stopping power of charged particles in the wide energy range for different materials have been continually measured until now [1-3]. Furthermore, some computer codes for its estimation have been developed and continuously corrected using available experimental data [4,5]. Among these codes, SRIM is most popular one and has been used by many researchers who need to know stopping powers of charged particles in certain material. The latest available version of this code is SRIM-2013.

In nuclear physics experiments, the different gases are usually used such as for the Parallel Plate Avalanche Counter (PPAC), Multi Sampling Ionization Chamber (MUSIC), active target, etc. $[6,7]$. For designing nuclear physics experiments using charged particles as well as for data analysis, the stopping power of charge particles in air and different gases are essential. For this purpose, the predicted values of SRIM code are used instead of experimental ones. This is due to a fact that not so many experimental data of stopping power are available. Once the predicted values were used, one should need to confirm their correctness. Therefore, the comparison between (C)2014 Vietnam Academy of Science and Technology 
calculated and experimental data of stopping power of the charged particles in used absorbers should be done.

A new 5SSD-2 Pelletron recently was installed at Hanoi University of Science [8]. The beams of $\alpha$-particle with energy up to $6 \mathrm{MeV}$ are available for doing research. Various nuclear physics experiments and application are planning. Stopping power of $\alpha$-particles in different materials including air and gases plays an important role for designing these future experiments. Therefore, we decided to measure this quantity experimentally.

Objective of this study is to determine experimentally the stopping powers of $\alpha$-particles emitted by a triple $\alpha$-source in the PR10 and isobutane gases which are usually used in our research.

\section{EXPERIMENTAL PROCEDURE}

Our measurement was done at the Research Center for Nuclear Physics (RCNP) of Osaka University (Japan). This is a part of our experiment for charge changing cross-section measurement which is to be performed in 2014 using RCNP RI beam. A triple alpha standard source of $9372 \mathrm{~Bq}$ consists of ${ }^{148} \mathrm{Gd},{ }^{241} \mathrm{Am}$ and ${ }^{244} \mathrm{Cm}$ isotopes produced by the company of Eckert \& Ziegler Isotope Products was used in this experiment. This composite source emits alpha particles with different energies. For our measurements, only three distinct energies of $3.2712 \mathrm{MeV}$ $\left({ }^{148} \mathrm{Gd}\right), 5.4857 \mathrm{MeV}\left({ }^{241} \mathrm{Am}\right)$ and $5.795 \mathrm{MeV}\left({ }^{244} \mathrm{Cm}\right)$ were used. In case of ${ }^{244} \mathrm{Cm}$, the difference between $5.7628 \mathrm{MeV}$ and $5.805 \mathrm{MeV}$ is only $42 \mathrm{keV}$. They can not be observed separately due to energy losses, straggling and finite resolution of spectrometer. Therefore the weighted mean energy of $5.795 \mathrm{MeV}$ for these two peaks was used. Using the branching ratio given in the table, a mean energy $5.795 \mathrm{MeV}$ of these two peaks was obtained. The experimental setup of our measurement is shown in Fig. 1.

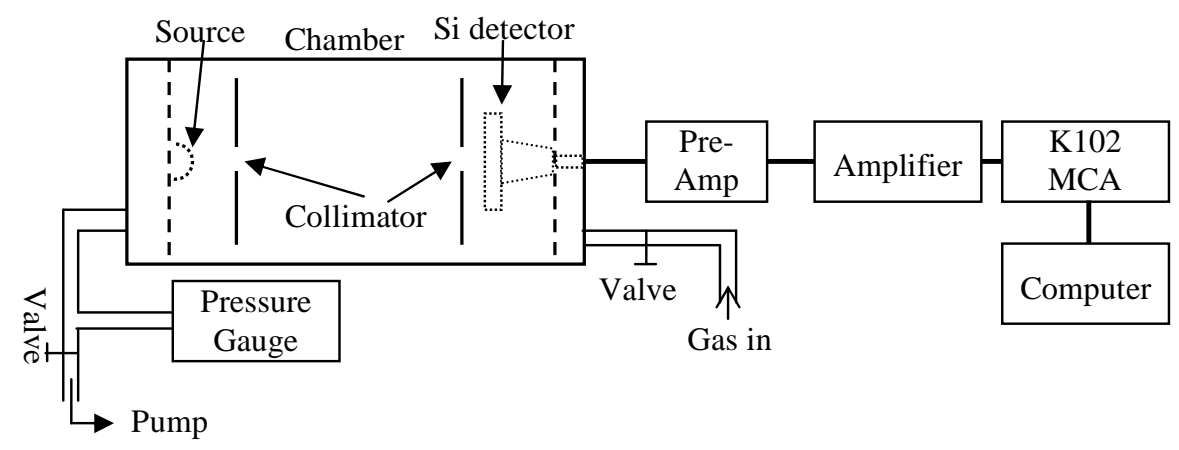

Fig. 1. Experimental setup.

A rectangular stainless steel vacuum chamber was used. The $\alpha$-source and a surface area silicon detector were fixed in the chamber during our measurements. The distance between the source and the detector was $31.7 \mathrm{~mm}$ and was remained constant throughout the experiment. The thickness of absorber between the source and the detector was changed by changing the pressure inside the chamber. Two aluminum plates of $2 \mathrm{~mm}$ thickness having the holes of $1 \mathrm{~mm}$ diameter 
were fixed along a line connected between the $\alpha$-source and the detector. They were used for collimation and for defining the beam direction.

The alpha particles pass through the gas under investigation and their residual energies after passing the investigated gas were measured by means of a surface barrier Si-detector of 300 micron thickness and $60 \mathrm{~mm} \times 90 \mathrm{~mm}$ dimension, which is connected to a spectrometer. The detector was mounted at the end of the chamber. The spectroscopy amplifier ORTEC451 and K102 USB based a 4096 channel analyzer of Kromak Company were used.

The commercial compressed gases of PR10 bought from Japan Air Gases Company and isobutane with purity of $99.995 \%$ and $99.990 \%$ bought from Sumitomo Seika Chemicals Company were used for the measurements. The errors came from impurities should be less than $0.01 \%$.

The vacuum system was evacuated to be better than $10^{-4}$ atm before filling with the gases under investigated. In addition, they were flushed several times before the final filling was introduced. Leakage of the system can be ignored during measurement. The gas pressure was measured with an absolute baratron manometer of MKS Company. The vacuum control system was connected with the vacuum chamber. The temperature of the chamber was fixed by an air conditioner in an experimental room.

To calibrate the spectrometer, the chamber was evacuated. The energy per channel was determined from the positions of the $3.2712 \mathrm{MeV}, 5.4857 \mathrm{MeV}$ and $5.795 \mathrm{MeV}$ peaks. The measured spectrum is shown in Fig. 2. The widths of these peaks were mainly due to the overall energy resolution of the spectrometer. Fig. 3 presents the energy calibration curve.

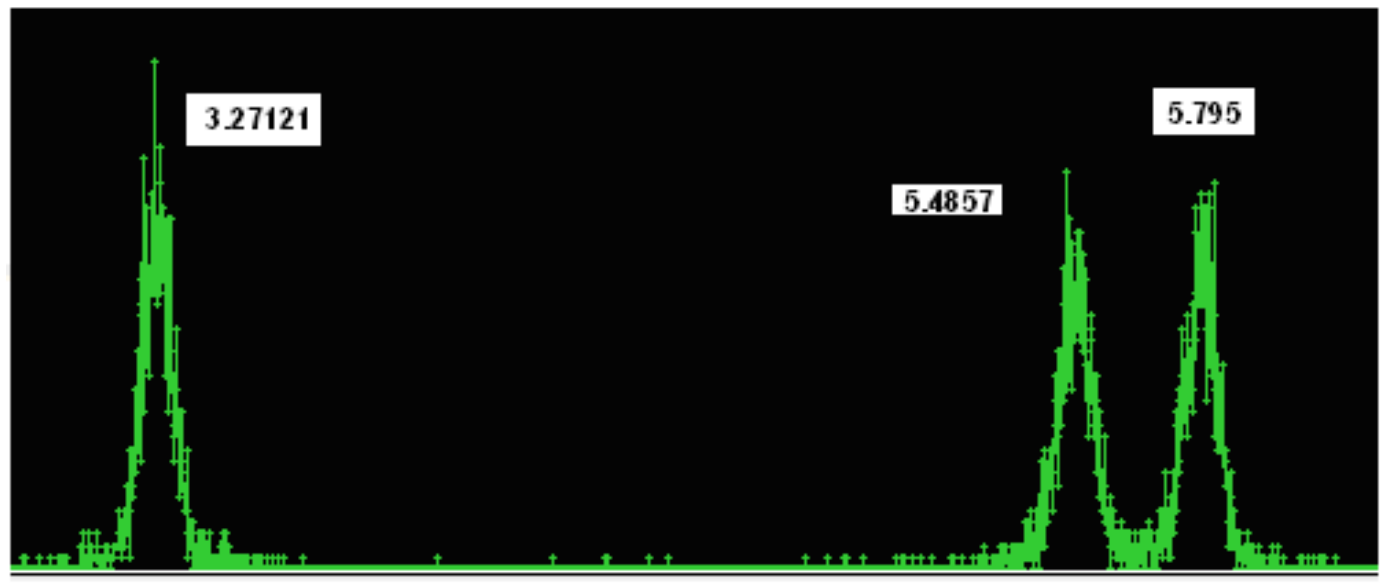

Fig. 2. The energy spectrum of the mixed alpha source.

Many residual-energy spectra have been taken for each gas at different pressures starting from $10^{-4}$ atm until the alpha particles fully stopped by the gas. These spectra were later analyzed for deducing the stopping power values.

\section{THE EXPERIMENTAL RESULTS}

The mean residual $\alpha$-particle energies were calculated from the center of the peaks in the pulse-height spectra. They were corrected for the energy losses within the gold dead layer on the 


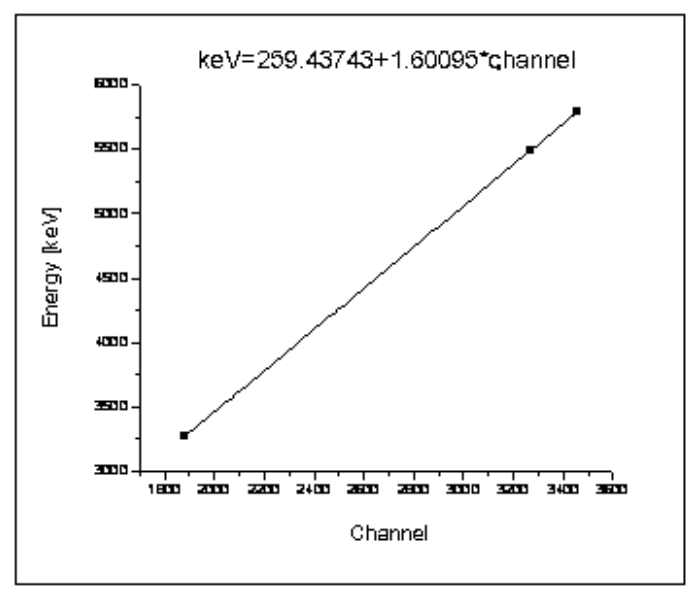

Fig. 3. The energy calibration curve of the spectrometer.

silicon detector which was $40 \mu \mathrm{g} / \mathrm{cm}^{2}$ thick. To that end the energy-loss data calculated by SRIM2013 code have been used. These corrections were not critical, because they amounted only up to $14 \mathrm{keV}$ at $1 \mathrm{MeV}$ and $9 \mathrm{keV}$ at $5 \mathrm{MeV}$. The energy losses within the source were negligible.

The finite source and aperture diameters caused some spread of the alpha-particle path lengths. The path-length distributions and the mean path lengths were computed by a Monte Carlo program. From the pressure and the temperature of the gas and from the mean path length, the traversed mass per unit area was calculated for each spectrum.

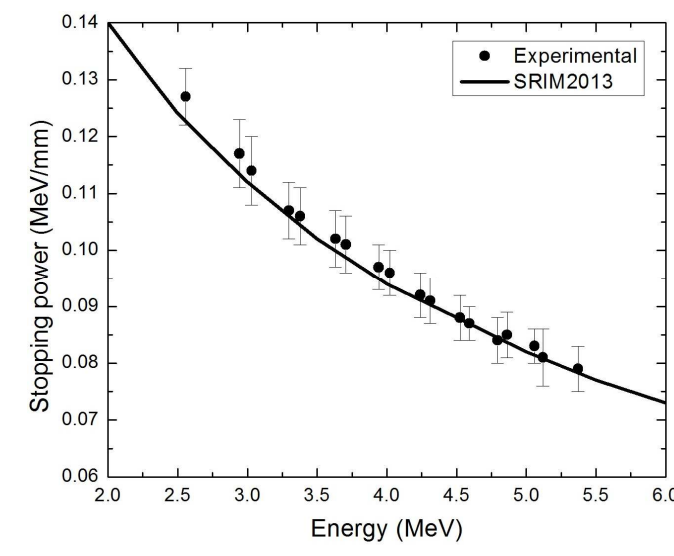

Fig. 4. Stopping Power of $\alpha$ particles in PR10 gas. Solid squares stand for experimental values while solid line represents the calculated values by SRIM2013.

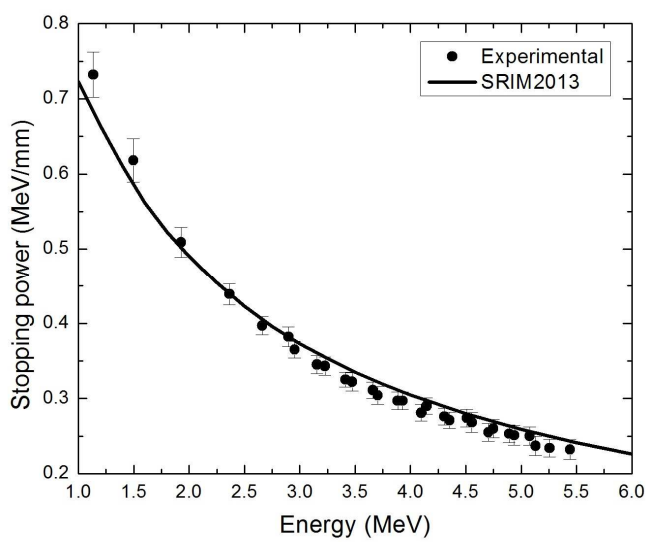

Fig. 5. Stopping Power of $\alpha$ particles in $\mathrm{C}_{4} \mathrm{H}_{10}$ gas. Solid squares stand for experimental values while solid line represents the calculated values by SRIM2013.

The corrected experimental data were fitted by least-squares polynomials representing the traversed gas mass per unit area as a function of the residual energy $\mathrm{E}$ within the interval from 
$0.5 \mathrm{MeV}$ to $5.3 \mathrm{MeV}$. The statistical errors of the residual energies obtained from the pulse-height spectra and the statistical errors of the traversed masses per unit area were taken into account.

For comparison, range curves were computed by SRIM-2013 computer program. The range curves are shown in Fig. 4 and Fig. 5. Our measured data agree rather well with the curves calculated by SRIM-2013.

\section{CONCLUSIONS}

The stopping powers up to $5.5 \mathrm{MeV}$ of $3.2712 \mathrm{MeV}\left({ }^{148} \mathrm{Gd}\right), 5.4857 \mathrm{MeV}\left({ }^{241} \mathrm{Am}\right)$ and $5.795 \mathrm{MeV}\left({ }^{244} \mathrm{Cm}\right)$ alpha particles in air, PR10 and iso-butane have been measured. The comparison of the experimental values with the predicted ones using SRIM-2013 code shows that they are in agree for PR10 and iso-butane gases while not for air. The experimental stopping powers of alpha particles in air are smaller than calculated ones in average about $6 \%$.

\section{ACKNOWLEDGMENTS}

This work is supported by Japan Society for the Promotion of Science (JSPS) and Vietnam Academy of Science and Technology (VAST) Joint Research Project.

\section{REFERENCES}

[1] J. F. Ziegler, J. P. Biersack, and U. Littmark In The Stopping and Range of Ions in Matter, Vol. 1, New York, 1985. Pergamon.

[2] P. Sigmund: Stopping of heavy ions Springer Tracts in Modern Physics 204 (2004).

[3] H. Bichsel, Stopping Power of Fast Charged Particles in Heavy Elements, National Institute of Standards and Technology, Report NIST IR-4550, 1991.

[4] J. F. Ziegler, SRIM-2013. Available from http://www.srim.org

[5] H. Paul and A. Schinner, Program MSTAR, version 1, Nucl. Instr.Meth. Phys. Res. B 179 (2001) 299, and version 2, Nucl. Instr. Meth. Phys. Res. B 195 (2002) 166.

[6] W. R. Leo, Techniques for Nuclear and Particle Physics Experiments, Springer, Berlin, Heidelberg, 1987.

[7] T. D. Trong, N. T. Tho, L. H. Khiem et al., Feasibility of Utilization of MUSIC for Particles Identification of Light Rich-neutron Isotopes, To be published in RCNP Annual Report.

[8] Nguyen The Nghia, Vi Ho Phong, Dinh Van Thin, Le Hong Khiem, Le Tuan Anh, Nguyen Van Quan, Bui Van Loat, Dong Van Thanh. VNU Journal of Science, Mathematics-Physics 28(1S) (2012) 109-113. 